\title{
Lactoferrin in Gingival Crevicular Fluid and Peripheral Blood during Experimental Gingivitis
}

\author{
Burcu Ozdemir \\ Gonen Ozcana \\ Burcu Karaduman ${ }^{a}$ \\ Aslı Idil Teoman ${ }^{\mathrm{b}}$ \\ Eylem Ayhan ${ }^{a}$ \\ Nazmi Ozer ${ }^{c}$ \\ Durdal Us ${ }^{\mathrm{d}}$
}

\section{ABSTRACT}

Objectives: Lactoferrin (LF) is an iron binding protein and stored in the specific granules of granulocytes. It is released by degranulation following granulocyte activation. A positive correlation was previously reported between periodontitis and LF titers of gingival crevicular fluid (GCF) and blood. The purpose of this study was to examine alterations of GCF and blood levels of LF (LF-GCF and LF-BL, respectively), employing the experimental gingivitis model.

Methods: Twelve systemically healthy volunteers, aged 19-21, were selected. Pre-experimental phase of hygiene was followed by a 14-day experimental gingivitis phase in which subjects refrained from all oral hygiene procedures. After that subjects resumed optimal plaque control for 21 days of recovery period. At days 0 (baseline), 14 and 35 gingival crevicular fluid (GCF) and blood samples were collected and plaque index (PI), gingival index (GI), bleeding on probing (BOP) and probing pocket depth scores were recorded. LF levels were measured with commercial enzyme-linked immunosorbent assay (ELISA) kit.

Results: PI, GI, BOP and LF-GCF increased significantly after 14 days of experimental gingivitis period and decreased significantly after reinstitution of oral hygiene measures $(P<.05)$. LF-BL appeared to follow the same pattern. Significant negative correlation was detected between the level of LF-BL and BOP at day $14(\mathrm{P}<.05)$, whereas significant positive correlation was noticed between LF$\mathrm{BL}$ and clinical scores $\mathrm{PI}, \mathrm{GI}$ and $\mathrm{BOP}$ at day $35(\mathrm{P}<.05)$.

Conclusions: LF-BL followed the same pattern with LF-GCF and clinical scores during the experimental gingivitis and recovery periods, although alterations of the LF-BL appeared statistically insignificant. (Eur J Dent 2009;3:16-23)

Key words: Lactoferrin; Gingival crevicular fluid; Gingivitis; Peripheral blood; Periodontal index.

a Department of Periodontology, Gazi University Faculty of Dentistry, Ankara, Turkey.

${ }^{b}$ Ministry of Health, Ankara, Turkey.

c Department of Biochemistry, Hacettepe University School of Medicine, Ankara, Turkey.

d Department of Microbiology, Hacettepe University School of Medicine, Ankara, Turkey.
Corresponding author: Dr. Burcu Ozdemir Gazi University, Faculty of Dentistry, Department of Periodontology, 84. Sokak, 8. Cadde, Emek, 06510, Ankara, Turkey. Email: cburcudagazi.edu.tr; burcucakilcidyahoo.com 


\section{INTRODUCTION}

The periodontal diseases has complex etiology and has been proposed that it is the host response to the long-term bacterial challenge, including increased neutrophil response and the release of proinflammatory mediators, which may be related with initiation and exacerbation of the systemic diseases and conditions. When compared to periodontally healthy subjects, significantly higher plasma white cell counts has been reported for subjects with gingivitis and periodontitis, and suggested that those high white cell counts might be related with systemic diseases such as myocardial infarction. ${ }^{1}$ Neutrophilic granulocytes play an important role at the primary defense of the host against pathogens and immunogenic materials. ${ }^{2}$ They are reported to be present in increased numbers within the gingival pocket and to be the predominant leukocytes in the pocket epithelium and adjacent connective tissue in periodontal diseases. ${ }^{3-5}$

Lactoferrin (LF), an iron binding protein with some antibacterial properties is stored in the specific (secondary) granule of the granulocytes and mainly released during migration and influences granulocyte functions such as adhesion and chemotaxis., ${ }^{3,6}$ LF has been proposed as a marker of the number of granulocytes. ${ }^{7}$ Following the activation of neutrophils, degranulation of specific granules with an instantaneous release of LF, has been demonstrated previously. ${ }^{6,7} \mathrm{LF}$ titers in various body fluids, including sputum and blood, were reported to be correlated with the presence of inflammation, such as periodontitis. ${ }^{8}$ Significantly higher levels of LF-GCF have been found at both periodontitis and gingivitis compared to healthy sites and those titers also reported to be degreased to the healthy sites' LF levels after periodontal treatment. 9,10

The human experimental gingivitis study model, introduced by Löe et al, ${ }^{4}$ has shown that qualitative effect of dental plaque created by total withdrawal of oral hygiene procedures leads to gingival inflammation and since this pioneering work of Löe et al, many studies have been undertaken using experimental gingivitis study model..$^{11-14}$

The purpose of our present study was to examine the alterations of LF levels in GCF and peripheral venous blood, resulting from the bacterial plaque accumulation in a healthy mouth, employing the experimental gingivitis model, in order to examine whether local gingival inflammation has some systemic influence.

\section{MATERIALS AND METHODS}

Patient selection

Twelve dental students attending Gazi University Faculty of Dentistry was recruited voluntarily. This participant group consisted of all systemically healthy males in age ranging from 19 to 21 years. They were selected on the presence of 24 teeth or more, absence of pockets $\geq 5 \mathrm{~mm}$ and absence of approximal attachment loss. ${ }^{15,16}$ No subject wore any orthodontic or prosthetic appliances. ${ }^{17}$ The use of any medication that might interfere with the outcome of the study, during the past 6 months, was an exclusion criterion, such as anti-inflammatory agents, antibiotics, and immunosuppressive drugs. All of the participants were non-smokers. The study protocol was approved by Institutional Review Board at Gazi University, School of Medicine and all subjects were asked to give an informed written consent to participate, after a thorough explanation of the procedures and objectives of the study.

\section{Experimental design}

Experimental design of the study consisted of 3 parts as pre-experimental, experimental and recovery periods (Figure 1 ):

- Pre-experimental period: Two weeks prior to the experimental period participants received a thorough dental prophylaxis and instruction in oral hygiene. During the following week professional tooth cleaning was repeated. Participants were regularly examined and their oral hygiene was monitored to achieve maximum gingival health. When participants had less than 20\% approximal bleeding sites, they entered a 14 day experimental gingivitis trial period. ${ }^{15,16}$

- Experimental period: Examiners made sure of the participants gingival health at the baseline of this period via clinical indices, then participants were led to enter this period by cessation of all plaque control measures. ${ }^{15,16}$ At day 0 (baseline) and 14 clinical parameters were recorded, and peripheral venous blood and GCF samples were taken. 
- Recovery period: After 14 day experimental period without any oral hygiene measures, participants received professional prophylaxis, instruction in oral hygiene and they were encouraged to return their daily oral hygiene measures. The professional tooth cleaning was repeated and periodontal condition regularly examined in order to achieve maximum gingival health during this 21 -day recovery period. ${ }^{5}$ At the end of the 21 day recovery period clinical indices were repeated and peripheral venous blood and GCF samples were taken.

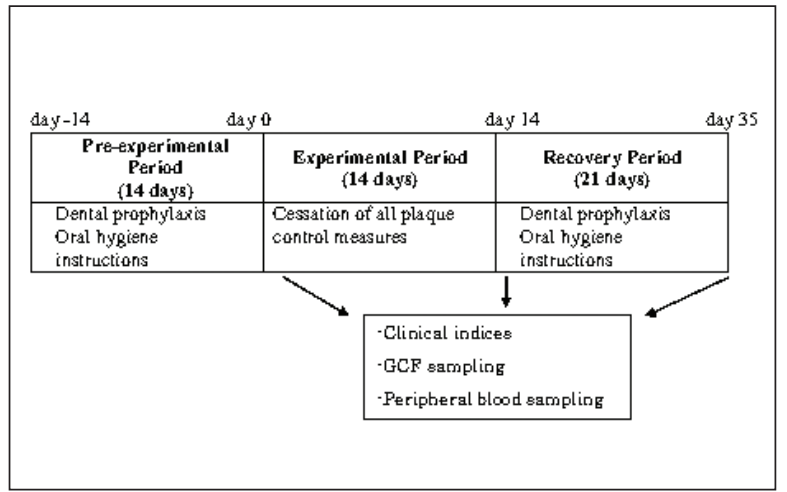

Figure 1. Experimental design of the study.

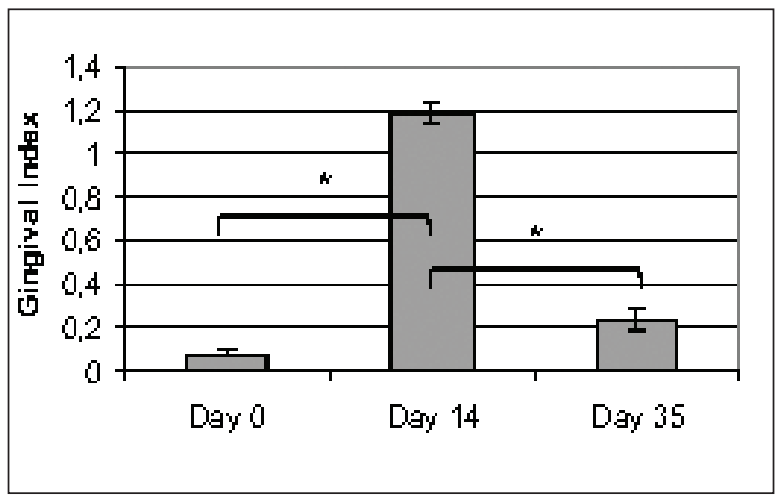

Figure 3. Gingival Index (*: $P<.05)$.

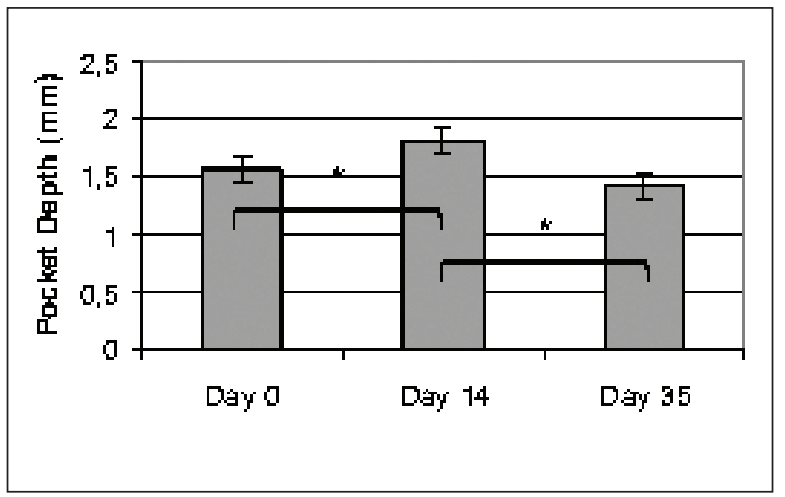

Figure 5. Pocket depth.
Periodontal examination

Following clinical parameters were recorded for monitoring the gingival situation of the participants before and during the experimental period and control period: Plaque index (PI), ${ }^{18}$ gingival index (GI), ${ }^{19}$ bleeding on probing $(\mathrm{BOP})^{20}$ and probing pocket depth (PD). All clinical parameters were measured with a William's probe calibrated in millimeters.

\section{GCF sampling}

For collection of GCF, prefabricated sterile paper strips (Periopaper ${ }^{\circledR}$ GCF Strips, Proflow, Amityville, NY) were used. The sites to be sampled

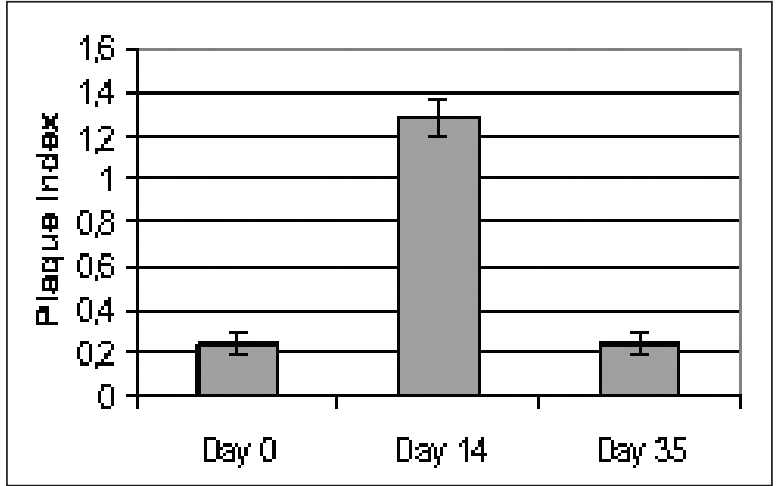

Figure 2. Plaque index $(*: P<.05)$.

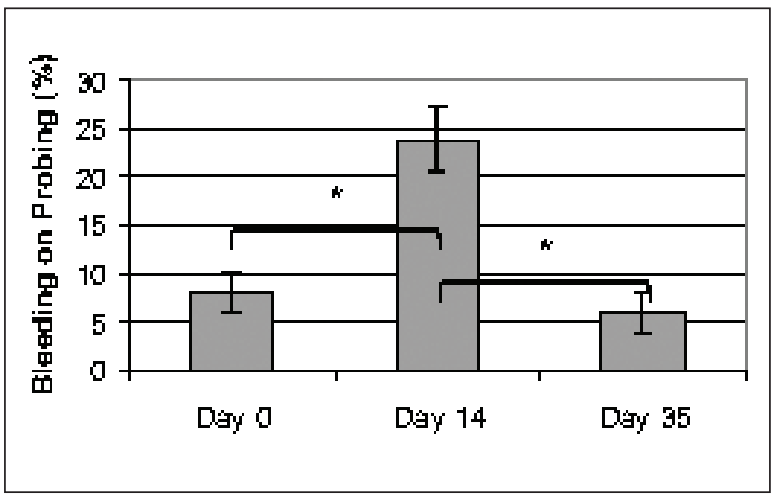

Figure 4. Bleeding on probing (*: $\mathrm{P}<.05)$.

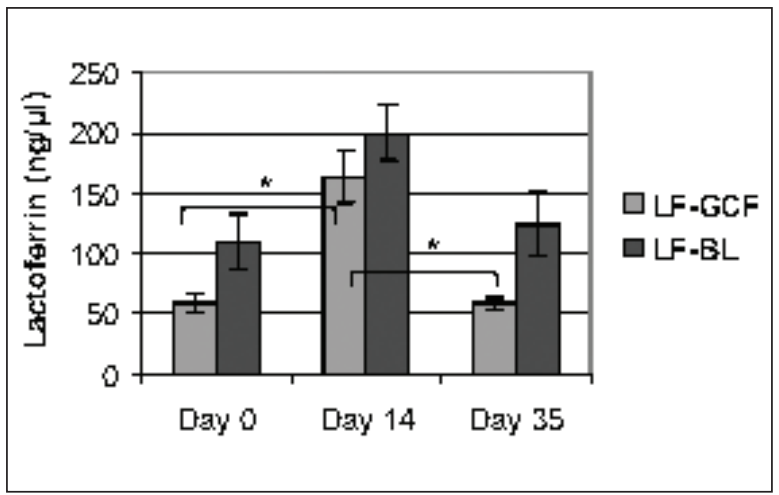

Figure 6. The levels of lactoferrin in GCF (LF-GCF) and blood (LF-BL) (*: P<.05). 
were isolated with cotton rolls and visible supragingival plaque was removed carefully by cotton pellets. The area was gently air-dried in an apico-coronal direction and $30 \mathrm{sec}$. later paper strips were inserted into the gingival crevice until mild resistance was felt and left there for $30 \mathrm{sec}{ }^{21}$ Strips contaminated by dental plaque, saliva, bleeding or exudates were discarded. Strips were stored in a labeled microcentrifuge tube and frozen at $-70^{\circ} \mathrm{C}$ until further processing. Prior to assaying strips were eluted into $300 \mu \mathrm{l}$ of $20 \mathrm{mM}$ phosphate buffer, pH 6.0, containing $0.15 \mathrm{M} \mathrm{NaCl}$ and $0.1 \%$ Tween $20 .{ }^{22}$

\section{Blood sampling}

Six milliliters of peripheral venous blood was drawn into glass test tubes that contain EDTA as an anticoagulant. After the separation of plasma, the samples were stored at $-70^{\circ} \mathrm{C}$ until further processing.

\section{Lactoferrin levels}

LF levels were determined by a commercial ELISA kit (Calbiochem, Darmstad, Germany) as described in the manufacturer's instructions. Different concentrations of LF $1100,50,25$, $12.5,6.2,3.1,1.6 \mathrm{ng} / \mu \mathrm{l}$ ) were prepared by the dilution of lyophilized LF standard, supplied by the manufacturer. The absorbance values (Optical Densities=0D) were measured spectrophotometrically at a wavelength of 450 $\mathrm{nm}$, and the results were evaluated quantitatively according to the ODs of standard LF concentrations. If the measured concentrations of LF in a sample were greater then $100 \mathrm{ng} / \mathrm{ml}$, the sample was retested by using further dilutions (1/100).

\section{Statistical analysis}

The clinical parameters, LF-GCF and LF$\mathrm{BL}$ were expressed as mean \pm standard error. Repeated measurement variance analysis (ANOVA) was used for assessment of the data obtained at day 0,14 and $35 .{ }^{23}$ The arc-sinus transformation was used for ANOVA assessments of BOP data. Bonferroni test was used for determination of different groups. The Pearson test was used for correlation assessments. ${ }^{23}$

\section{RESULTS}

Clinical parameters

The mean clinical parameters and their standard errors are summarized in Figures 2-5. PI, $\mathrm{GI}$ and BOP scores showed statistically significant increase during the experimental period of 14 days and significant decrease thereafter, as expected $(P<.01)$. Pearson correlation and $P$ values are summarized at Tables 1 and 2.

\section{Lactoferrin levels}

The mean data of LF levels ( \pm standard errors) in GCF (LF-GCF) and blood (LF-BL) and their standard deviations are summarized at Figure 6.

The differences between mean LF-GCF levels for time intervals found to be statistically significant $(P<$.01). From day 0 to day 14 , mean LF-GCF levels significantly increased $(58.8 \pm 7.2$ and $163.2 \pm 21.9$ $\mathrm{ng} / \mu \mathrm{l}$, respectively) (Min: $29 \mathrm{ng} / \mu \mathrm{l}$, Max: $98 \mathrm{ng} / \mu \mathrm{l}$ and min: $72 \mathrm{ng} / \mu \mathrm{l}$, max: $297 \mathrm{ng} / \mu \mathrm{l}$, respectively) $(P<.05)$, and significantly decreased at day 35 (58.7 $\pm 5.4 \mathrm{ng} / \mu \mathrm{l}$ ) (Min: $26 \mathrm{ng} / \mu \mathrm{l}$, Max: $191 \mathrm{ng} / \mu \mathrm{l}$ ) $(P<.05)$. The pattern of LF-GCF levels followed during all of the periods of the study appeared to be similar and compatible with pattern of $\mathrm{PI}, \mathrm{GI}$ and BOP scores, although no statistically significant correlation was detected (Tables 1 and 2).

The mean level of LF-BL at day 0 which was detected as $112.0 \pm 23.4 \mathrm{ng} / \mu \mathrm{l}$ (Min: $1 \mathrm{ng} / \mu \mathrm{l}$, Max: $255 \mathrm{ng} / \mu \mathrm{l}$ ), was increased to $200.0 \pm 22.9 \mathrm{ng} / \mu \mathrm{l}$ (Min: $7 \mathrm{ng} / \mu \mathrm{l}$, Max: $289 \mathrm{ng} / \mu \mathrm{ll}$ at day 14 and decreased to $125.0 \pm 27.5 \mathrm{ng} / \mu \mathrm{l}$ (Min: $9 \mathrm{ng} / \mu \mathrm{l}$, Max: $256 \mathrm{ng} / \mu \mathrm{l}$ ) at day 35 . No statistically significant differences were found between the time intervals. Levels of LF-BL demonstrated almost the same pattern with LF-GCF, PI, GI and BOP at days 0, 14 and 35. However, a significant negative correlation existed between the level of LF-BL and BOP at day 14 $(P<.05)$, whereas significant positive correlation existed between the level of LF-BL and scores of $\mathrm{PI}, \mathrm{GI}$ and BOP at day $35(\mathrm{P}<.05)$ (Tables 1 and 2).

\section{DISCUSSION}

In the present study it was aimed to examine previously hypothesized systemic inflammatory response triggered by dental plaque accumulation via assessment of neutrophil protease LF levels within GCF and peripheral venous blood, employing the experimental gingivitis model. Kowolik et $\mathrm{al}^{5}$ demonstrated that circulating neutrophils were 
gradually increased during the experimental gingivitis period of 21 days and they stated that results of their study support the hypothesis that the accumulation of dental plaque can result in a measurable systemic inflammatory response.

The present study, intentionally, consisted of solely male, systemically healthy and non-smoker dental student volunteers of the same age group. It has been reported that hormonal influences caused by gender differences and age may alter the response to the bacterial challenge. 5,24,25 Smoking has previously been associated with etiology and pathogenesis of the periodontal diseases and also with the outcomes of the periodontal treatments. ${ }^{26-}$ ${ }^{28}$ Smoking has also been reported to have adverse effects on PMN viability and function within GCF of periodontally healthy population. ${ }^{29}$
Human experimental gingivitis, which was first introduced almost half century ago, is still one of the most preferred model for researchers to investigate the nature of periodontal diseases..$^{24,30}$ The clinical scores of the present study was consistent with the previous studies recruiting the experimental study model. ${ }^{13,16}$

In GCF, significantly higher levels of LF have been found at both periodontitis and gingivitis sites compared to healthy sites. ${ }^{9}$ On the other hand, Gustafsson et $\mathrm{al}^{3}$ reported that the LFGCF did not differ significantly between gingivitis, periodontitis with/without destruction and authors claimed that LF-GCF levels were not related to the local severity of the periodontal disease.

In our present study, LF-GCF increased significantly during the course of the plaque

Table 1. Pearson correlation between PI, PPD, GI, BOP, LF-BL AND LF-GCF at day-14.

\begin{tabular}{|c|c|c|c|c|c|}
\hline & PI & PPD & GI & BOP & LF-BL \\
\hline \multirow{2}{*}{ PPD } & $0.241 *$ & & & & \\
\hline & $0.451+$ & & & & \\
\hline \multirow{2}{*}{ GI } & 0.334 & 0.290 & & & \\
\hline & 0.289 & 0.361 & & & \\
\hline \multirow{2}{*}{ BOP } & 0.361 & 0.189 & 0.790 & & \\
\hline & 0.250 & 0.557 & 0.002 & & \\
\hline \multirow{2}{*}{ LF-BL } & -0.243 & -0.169 & -0.502 & -0.730 & \\
\hline & 0.447 & 0.599 & 0.097 & 0.007 & \\
\hline \multirow{2}{*}{ LF-GCF } & -0.032 & 0.318 & 0.146 & -0.064 & 0.390 \\
\hline & 0.922 & 0.314 & 0.651 & 0.843 & 0.210 \\
\hline
\end{tabular}

* : Pearson correlation $(r)$.

† : P value.

Table 2. Pearson correlation between PI, PPD, GI, BOP, LF-BL AND LF-GCF at day-35.

\begin{tabular}{|c|c|c|c|c|c|}
\hline & $\mathrm{PI}$ & PPD & $\mathrm{GI}$ & BOP & LF-BL \\
\hline \multirow{2}{*}{ PPD } & $0.377^{*}$ & & & & \\
\hline & $0.227 \dagger$ & & & & \\
\hline \multirow{2}{*}{ GI } & 0.593 & 0.492 & & & \\
\hline & 0.042 & 0.104 & & & \\
\hline \multirow{2}{*}{ BOP } & 0.353 & 0.562 & 0.610 & & \\
\hline & 0.260 & 0.057 & 0.035 & & \\
\hline \multirow{2}{*}{ LF-BL } & 0.630 & 0.543 & 0.608 & 0.650 & \\
\hline & 0.028 & 0.068 & 0.036 & 0.022 & \\
\hline \multirow{2}{*}{ LF-GCF } & -0.152 & -0.217 & -0.272 & -0.541 & -0.517 \\
\hline & 0.638 & 0.498 & 0.392 & 0.069 & 0.086 \\
\hline
\end{tabular}

$*$ : Pearson correlation $(r)$.

† : P value. 
accumulation period which is in agreement with the findings of Fransson et al. ${ }^{24}$ In that study of Fransson et $\mathrm{al}^{2}{ }^{24}$ a very similar protocol, with a similar age group (aged 20-25years) of participants, was followed as in our present study; however the experimental period was longer (21 days). A strong agreement was found between the two studies by the means of LF-GCF increase from day 0 to $14^{\text {th }}$ day of the experimental gingivitis period. Fransson et $\mathrm{al}^{24}$ reported that LF-GCF increased 2,2 times after 14 days of plaque accumulation $(125 \mathrm{ng} / \mu \mathrm{l}$ to $277 \mathrm{ng} / \mu \mathrm{l}$, which is quite close to our findings that indicate 2.8 times increase of LF-GCF from day 0 to $14(58.8 \pm 7.2 \mathrm{ng} / \mu \mathrm{l}$ to $163.2 \pm 21.9 \mathrm{ng} / \mu \mathrm{l}) .{ }^{24}$

The results of this study also showed that GCF-LF levels decreased significantly after the reinstitution of oral hygiene measures and approached the levels that observed at baseline day 0 . This probably reflects the decreasing of neutrophil chemotactic factors in the crevice following plaque elimination and resolution of inflammation. This is in agreement with the results of an earlier study by Adonogianaki et al. ${ }^{31}$ Authors examined four acute-phase proteins, including LF, in GCF during the course of a 21 day experimental gingivitis study and reported that the concentrations of LF were significantly elevated during the course of the trial and when plaque control was reestablished these values declined. It has also been suggested previously that LFGCF might be a suitable marker for monitoring of treatment effects in periodontal disease, particularly periodontitis. ${ }^{32,33}$

Previous studies have pointed out a positive correlation between periodontitis and LF titers in GCF and blood. $8,10 \mathrm{LF}$ is known as a prominent component of PMNs and is released in infected tissues and blood during the inflammatory process. Suomalainen et $\mathrm{al}^{10}$ found increased amounts of LF in GCF and peripheral blood PMNs, in localized juvenile periodontitis. Furthermore, lower levels of LF was found in periodontitis sites during periodontal therapy, and this was followed by a rapid resumption to normal levels observed in healthy controls. In another study conducted by Martins et $a l,{ }^{8}$ the results showed that LF titers in sputum, gingival swabs, and blood correlate with the presence of inflammation. Although there are some data about plasma levels of LF at periodontitis, our present study appears to be the first one to examine plasma levels of LF during the experimental gingivitis.

In the present study, our data showed that LFBL increased from day 0 to 14 , decreased from day 14 to 35 , and finally reached almost the baseline values at day 35 , the end of the recovery period. Although statistical analysis failed to detect significant positive correlation between clinical scores or LF-GCF and LF-BL at days 0 and 14, still the patterns of LF-BL seemed to be compatible with LF-GCF, PI, GI and BOP scores at baseline, end of the experimental gingivitis and recovery periods. Significant negative correlation $(P<.05)$ was detected at day 14 between LF-BL and BOP. On the other hand, significant positive correlation existed between LF-BL and PI, GI and BOP at day $35(P<.05)$ which suggested that recovery from gingivitis has significant influences on LF-BL.

The pattern of neutrophil protease LF within plasma might simply be associated with momentary release of LF from the specific granules of activated neutrophils by local bacterial insult, such as the pattern of LF-GCF levels. ${ }^{6,7}$ On the other hand, insignificance of plasma LF level alteration during the experimental and recovery period might be related with common description of gingivitis as "a local phenomenon" in which migration of neutrophils into the oral cavity, via the gingival crevicular fluid increases, as part of the inflammatory response. This process provides the first line of cellular host defence to localize the oral flora and possibly reduce the likelihood of systemic spread. Moreover, it can be claimed that both statistically insignificant plasma and significant LF-GCF alterations during the same time courses, considered with likeness of their increasing and decreasing patterns, might be attributed to the effectiveness of the first line of host defence for avoiding systemic spread in systemically healthy individuals.

In addition, it is known that serum levels of neutrophil proteases, such as LF and PMNelastase, have been associated with several mild to moderate inflammatory conditions, such as male genital track inflammation and Helicobacter pylori infection. ${ }^{34-36}$ It was demonstrated that LF serum levels boost up to higher levels within 30-120 min of intravenous infusion of lipopolysaccharide or alive Escherichia coli, in vivo. ${ }^{37}$ Furthermore, not only bacterial contamination, also intense 
physical exercise and stress shown to be effective on induction of neutrophil activation, as manifested by release of protease content of neutrophil granules. ${ }^{38,39}$ Even if systemic health was one of the inclusion criteria of our study, still LF-BL might be affected from existence of minor undiagnosed inflammations or even from intense physical exercise.

\section{CONCLUSIONS}

In conclusion, the data of the present study demonstrated that the local inflammation created by plaque accumulation during the experimental gingivitis model caused elevated LF levels of GCF and blood. Furthermore, the treatment of local inflammation and returning to daily oral hygiene procedures caused reduction of LF levels in both GCF and BL. However, only LF-GCF alterations were appeared to be statistically significant, in this short term study. Within the limits of this study, our present data contributes to the hypothesis that not only periodontitis, also local microbial dental plaque accumulation and so caused local gingival inflammation might have systemic influences by means of LF release from activated neutrophils. Moreover, further investigations will help better understanding of the possible extents of the potential systemic effects of gingivitis.

\section{ACKNOWLEDGEMENTS}

Authors gratefully acknowledge Fikret Gurbuz, Professor, Ankara University, Faculty of Agriculture, Animal Science Department, Biometry and Genetic Discipline, for his contribution in statistical analyses of the data of the present study.

This study was funded by Gazi University Research Grant 03/2002-30.

\section{REFERENCES}

1. Kweider M, Lowe GD, Murray GD, Kinane DF, McGowan DA. Dental disease, fibrinogen and white cell count; links with myocardial infarction? Scott Med J 1993;38:73-74.

2. Cassatella MA. Neutrophil: An Emerging Regulator of Inflammatory and Immune Response. Basel, Switzerland: Karger Publishers.; 2003.

3. Gustafsson A, Asman B, Bergstrom K. Elastase and lactoferrin in gingival crevicular fluid: possible indicators of a granulocyte-associated specific host response. $J$ Periodontal Res 1994;29:276-282.
4. Christersson LA, Albini B, Zambon JJ, Wikesjo UM, Genco RJ. Tissue localization of Actinobacillus actinomycetemcomitans in human periodontitis. I. Light, immunofluorescence and electron microscopic studies. $J$ Periodontol 1987;58:529-539.

5. Kowolik MJ, Dowsett SA, Rodriguez J, De La Rosa RM, Eckert GJ. Systemic neutrophil response resulting from dental plaque accumulation. J Periodontol 2001;72:146151.

6. Bentwood BJ, Henson PM. The sequential release of granule constitutents from human neutrophils. $J$ Immunol 1980;124:855-862.

7. Wright DG, Gallin JI. Secretory responses of human neutrophils: exocytosis of specific (secondary) granules by human neutrophils during adherence in vitro and during exudation in vivo. J Immunol 1979;123:285-294.

8. Martins CA, Fonteles MG, Barrett LJ, Guerrant RL. Correlation of lactoferrin with neutrophilic inflammation in body fluids. Clin Diagn Lab Immunol 1995;2:763-765.

9. Adonogianaki E, Moughal NA, Kinane DF. Lactoferrin in the gingival crevice as a marker of polymorphonuclear leucocytes in periodontal diseases. $J$ Clin Periodontol 1993;20:26-31.

10. Suomalainen K, Saxen L, Vilja P, Tenovuo J. Peroxidases, lactoferrin and lysozyme in peripheral blood neutrophils, gingival crevicular fluid and whole saliva of patients with localized juvenile periodontitis. Oral Dis 1996;2:129-134.

11. Gaumer HR, Holm-Pedersen P, Folke LE. Indirect blastogenesis of peripheral blood leukocytes in experimental gingivitis. Infect Immun 1976;13:1347-1353.

12. Seymour GJ, Gemmell E, Walsh LJ, Powell RN. Immunohistological analysis of experimental gingivitis in humans. Clin Exp Immunol 1988;71:132-137.

13. Preshaw PM, Knutsen MA, Mariotti A. Experimental gingivitis in women using oral contraceptives. J Dent Res 2001;80:2011-2015.

14. Trombelli L, Farina R, Manfrini R, Tatakis DN. Modulation of clinical expression of plaque-induced gingivitis: effect of incisor crown form. J Dent Res 2004;83:728-731.

15. Lie MA, Timmerman MF, van der Velden U, van der Weijden GA. Evaluation of 2 methods to assess gingival bleeding in smokers and non-smokers in natural and experimental gingivitis. J Clin Periodontol 1998;25:695-700.

16. Lie MA, van der Weijden GA, Timmerman MF, Loos BG, van Steenbergen TJ, van der Velden U. Oral microbiota in smokers and non-smokers in natural and experimentallyinduced gingivitis. J Clin Periodontol 1998;25:677-686.

17. Daly CG, Highfield JE. Effect of localized experimental gingivitis on early supragingival plaque accumulation. $J$ Clin Periodontol 1996;23:160-164. 
18. Silness J, Löe H. Periodontal disease in pregnancy. II. Correlation between oral hygiene and periodontal condition. Acta Odontol Scand 1964;22:121-135.

19. Löe $\mathrm{H}$, Silness J. Periodontal disease in pregnancy. I.Prevalence and severity. Acta Odontol Scand 1963;21:533551.

20. Muhlemann HR, Son S. Gingival sulcus bleeding-a leading symptom in initial gingivitis. Helv Odontol Acta 1971;15:107113.

21. Tuter G, Kurtis B, Serdar M. Interleukin-1beta and thiobarbituric acid reactive substance (TBARS) levels after phase I periodontal therapy in patients with chronic periodontitis. J Periodontol 2001;72: 883-888.

22. Chen HY, Cox SW, Eley BM, Mantyla P, Ronka H, Sorsa T. Matrix metalloproteinase-8 levels and elastase activities in gingival crevicular fluid from chronic adult periodontitis patients. J Clin Periodontol 2000;27:366-369.

23. Wei PF, Ho KY, Ho YP, Wu YM, Yang YH, Tsai CC. The investigation of glutathione peroxidase, lactoferrin, myeloperoxidase and interleukin-1beta in gingival crevicular fluid: implications for oxidative stress in human periodontal diseases. J Periodontal Res 2004;39:287-293.

24. Fransson C, Mooney J, Kinane DF, Berglundh T. Differences in the inflammatory response in young and old human subjects during the course of experimental gingivitis. J Clin Periodontol 1999;26:453-460.

25. Matheny JL, Abrams H, Johnson DT, Roth GI. Microcirculatory dynamics in experimental human gingivitis. J Clin Periodontol 1993;20:578-583.

26. Preber $\mathrm{H}$, Linder L, Bergstrom J. Periodontal healing and periopathogenic microflora in smokers and non-smokers. J Clin Periodontol 1995;22:946-952.

27. Stavropoulos A, Mardas N, Herrero F, Karring T. Smoking affects the outcome of guided tissue regeneration with bioresorbable membranes: a retrospective analysis of intrabony defects. J Clin Periodontol 2004;31:945-950.

28. Feldman RS, Bravacos JS, Rose CL. Association between smoking different tobacco products and periodontal disease indexes. J Periodontol 1983;54:481-487.

29. Guntsch A, Erler M, Preshaw PM, Sigusch BW, Klinger G, Glockmann E. Effect of smoking on crevicular polymorphonuclear neutrophil function in periodontally healthy subjects. J Periodontal Res 2006;41:184-188.

30. Giannopoulou C, Andersen E, Demeurisse C, Cimasoni G. Neutrophil elastase and its inhibitors in human gingival crevicular fluid during experimental gingivitis. $J$ Dent Res 1992;71:359-363.
31. Adonogianaki E, Moughal NA, Mooney J, Stirrups DR, Kinane DF. Acute-phase proteins in gingival crevicular fluid during experimentally induced gingivitis. $J$ Periodontal Res 1994;29:196-202.

32. Jentsch H, Sievert Y, Gocke R. Lactoferrin and other markers from gingival crevicular fluid and saliva before and after periodontal treatment. J Clin Periodontol 2004;31:511514.

33. Tsai CC, Kao CC, Chen CC. Gingival crevicular fluid lactoferrin levels in adult periodontitis patients. Aust Dent $J$ 1998;43:40-44.

34. Rocha-Pereira P, Santos-Silva A, Rebelo I, Figueiredo A, Quintanilha A, Teixeira $F$. The inflammatory response in mild and in severe psoriasis. Br J Dermatol 2004;150:917928.

35. Racz I. Immunological aspects of Helicobacter pylori infection. Acta Physiol Hung 2000;87:329-337.

36. Kopa Z, Wenzel J, Papp GK, Haidl G. Role of granulocyte elastase and interleukin- 6 in the diagnosis of male genital tract inflammation. Andrologia 2005;37:188-194.

37. Gutteberg TJ, Rokke O, Andersen O, Jorgensen T. Early fall of circulating iron and rapid rise of lactoferrin in septicemia and endotoxemia: an early defence mechanism. Scand $J$ Infect Dis 1989;21:709-715.

38. Gray AB, Telford RD, Collins M, Baker MS, Weidemann MJ. Granulocyte activation induced by intense interval running. J Leukoc Biol 1993;53:591-597.

39. Cannon JG, Angel JB, Abad LW, O'Grady J, Lundgren N, Fagioli L, Komaroff AL. Hormonal influences on stressinduced neutrophil mobilization in health and chronic fatigue syndrome. J Clin Immunol 1998;18:291-298. 